\title{
Sex-related differences of cAMP specific PDE4B3 mRNA in oligodendrocytes following systemic inflammation
}

\author{
Emily M Johansson, Cristina Sanabra and Guadalupe Mengod \\ Departament de Neuroquímica i Neurofarmacologia, Institut d'Investigacions Biomèdiques de \\ Barcelona (CSIC), IDIBAPS, CIBERNED, Rosselló, 161, 08036 Barcelona, Spain
}

Running title: Sex differences in PDE4B mRNAs following LPS

Key words: neuroinflammation, LPS, in situ hybridization, multiple sclerosis, ICER

Corresponding author: Guadalupe Mengod; Department of Neurochemistry and Neuropharmacology, Institut d'Investigacions Biomèdiques de Barcelona, CSIC- IDIBAPS, Rosselló, 161, 08036 Barcelona, Spain. Phone: +3493-363 8323; Fax: +3493-363 8301; E-mail: guadalupe.mengod@iibb.csic.es

\begin{abstract}
Sex-related differences have been observed in the incidence and severity of several neurological diseases and in sepsis in humans. Cyclic adenosine monophosphate (cAMP) has been shown to play an important role in modulating the inflammatory environment during neuroinflammation and importantly in protecting myelin from excitotoxic cell death. Considering the sexual dimorphism in the functional properties of oligodendrocytes and the importance of a systemic inflammation in the progression of multiple sclerosis, we focused on identifying possible sex-related differences in the alterations previously reported for the two phosphodiesterase4B (PDE4B) splice-variants (PDE4B2 and PDE4B3) mRNA expression during innate neuroinflammation. PDE4A, PDE4B and PDE4D are present in oligodendrocytes and we have previously reported that PDE4B3 mRNA is readily expressed in both oligodendrocytes and neurons.

In the present study we analyzed the influence of an intra-peritoneal lipopolysaccharide injection on the distribution pattern and expression levels of the PDE4B mRNA splicing variants in both male and female mice brains. Clear differences were observed in PDE4B2 and PDE4B3 mRNA expression levels in males compared to females in a time-dependent manner. Furthermore, we observed that the clear downregulation of PDE4B3 mRNA was reflected in a lower percentage of oligodendrocytes positive for this transcript which correlated with a decrease in inducible cAMP early repressor (ICER) expression in female corpus callosum.
\end{abstract}




\section{Introduction}

Sex-related differences in susceptibility and nature are observed in several neurological diseases e.g. Alzheimer's disease, schizophrenia, depression, stroke, autism and autoimmune diseases, like multiple sclerosis (MS) and systemic lupus erythematosus (for reviews see (Czlonkowska et al., 2005;Darnall and Suarez, 2009). Approximately $78 \%$ of people affected by autoimmune diseases are women (Fairweather et al., 2008). Moreover, during innate inflammation, women normally respond by more vigorous cellular reaction (for review see (Ansar et al., 1985), compared to men who react with higher proinflammatory cytokine production, elevating the severity of the innate inflammation (Marriott et al., 2006). Differences have also been observed in the incidence and severity of sepsis in humans (Schroder et al., 1998).

The proinflammatory agent lipopolysaccharide (LPS) is a potent activator of the innate immune system. LPS provokes stimulation of complex intracellular signaling pathways activating different proinflammatory transcription factors like cyclic adenosine monophosphate (CAMP) response element-binding (CREB), activator protein 1(AP-1) and nuclear factor kappa (Kawai et al., 2001; Lu et al., 2008). Sex-related differences are observed in the cytokine response towards LPS administration in rodents (Ashdown et al., 2007; Marriott et al., 2006).

CAMP is involved in the control of the acute inflammatory response, preventing progression to a non-beneficial chronic state (for review see (Moore and Willoughby, 1995). cAMP levels are regulated by a balance between the activities of two enzymes: adenylyl cyclase and cyclic nucleotide phosphodiesterase (PDE) and pharmacological manipulation using specific PDE inhibitors, in particular, PDE4 inhibitors provoke profound anti-inflammatory responses (for review see (Banner and Trevethick, 2004).

PDE4 together with PDE7 and PDE8 specifically hydrolyze CAMP in mammals. There is a large variety of different variants and splicing forms from the genes (Bender and Beavo, 2006) with overlapping properties but with differences in functionality and anatomical distribution (Conti, 2000; Reyes-Irisarri et al., 2008;Pérez-Torres et al., 2000). These characteristics, together with intracellular compartmentalization (Arp et al., 2003) provide several possibilities for selective therapeutic targeting.

CAMP-dependent activation of the CREB cascade initiates transcription of PDE4 (D'Sa et al., 2002). Especially noteworthy is that LPS phosphorylates CREB in a CAMP- independent manner without induction of CREB-dependent transcription (Avni et al., 2010). On the other hand, inducible cAMP early repressor (ICER), a potent endogenous repressor of cAMP response element (CRE) -mediated gene transcription, can be induced by a variety of stimuli like stress, seizure and LPS (for review see (Borlikova and Endo, 2009).

The alterations of the PDE4B mRNA splice variants in rodent brain in animal models of innate neuroinflammation (Johansson et al., 2011) and MS (Reyes-Irisarri et al., 2007) Sanabra, Johansson and Mengod, submitted, 2012), combined with both the role of cAMP in protecting myelin from excitotoxic cell death (Yoshioka et al., 1998), and the sexual dimorphism in neurological diseases prompted us to examine sex-related differences in the PDE4B mRNA splice variant expression following LPS administration. We speculate that these enzymes may play a role during innate neuroinflammation and consequently also during the initiation and relapses of experimental autoimmune encephalomyelitis an animal model of MS by affecting the inflammatory environment (Moreno et al., 2011).

In the present work, we found sex-related differences in the alterations for both PDE4B2 and PDE4B3 mRNAs expression in a time-dependent manner. We also show a significant difference between male and female in the effect of LPS on the PDE4B3 splice variant mRNA in oligodendrocytes correlating with alterations in ICER expression in female. 


\section{Material and Methods}

\section{Lipopolysaccaride administration}

Adult male and female C57BL6 mice (15-20g) six week old were purchased from Charles River Laboratories (France). All experimental procedures followed the European Communities Council Directive of November 24, 1986 (86/609/EEC), and were approved by the ethics committee of the University of Barcelona and of the Generalitat de Catalunya. Every effort was made to minimize the number of animals used and their suffering. The mice were maintained on a 12-h light/dark cycle at a constant environmental temperature with free access to food and water for one week prior to experimentation.

A dose-response curve for PDE4B3 mRNA expression was obtained using the following doses of LPS in female mice (serotype 055:B5, Sigma-Aldrich, Steinheim, Germany): 0.1, 0.3, 1, 5, 10 $\mathrm{mg} / \mathrm{kg}(\mathrm{n}=3 /$ dose) dissolved in sterile $0.9 \% \mathrm{NaCl}$. A dose-response curve was previously obtained in male (Johansson et al., 2011). Based on these curves and in order to obtain unambiguous mRNA expression for double in situ hybridization experiments, $10 \mathrm{mg} / \mathrm{kg}$ bacterial LPS was subsequently administered by an intraperitoneal (i.p.) injection. For biochemical experiments the lower dose $(5 \mathrm{mg} / \mathrm{kg}$ ) were used. Male mice were euthanized by cervical dislocation at $1 \mathrm{~h}, 3 \mathrm{~h}, 8 \mathrm{~h}$ and $24 \mathrm{~h}$ ( $\mathrm{n}=5$ /time point) after injection for histochemical studies and was further compared with previous results (Johansson et al., 2011); at $1 \mathrm{~h}, 3 \mathrm{~h}, 16 \mathrm{~h}$ and $24 \mathrm{~h}$ ( $\mathrm{n}=6-10 /$ time point) after injection, from two separate experiments, for biochemistry or at $1 \mathrm{~h}, 3 \mathrm{~h}, 8 \mathrm{~h}, 24 \mathrm{~h}$ and $32 \mathrm{~h}$ ( $\mathrm{n}=3-8 /$ time point) after injection, from two separate experiments, for cAMP assay. Female mice were euthanized at $1 \mathrm{~h}, 3 \mathrm{~h}, 8 \mathrm{~h}, 24 \mathrm{~h}$ and $48 \mathrm{~h}$ ( $n=8 /$ time point) after injection, from two separate experiments, for histochemistry; and for biochemistry and CAMP assay at the same way as reported for male. Controls were made to evaluate the effect of the injection with $\mathrm{NaCl}$ alone. In the first experiment euthanasia of female control-mice were made at $1 \mathrm{~h}, 3 \mathrm{~h}, 8 \mathrm{~h}, 24 \mathrm{~h}$ and $48 \mathrm{~h}(\mathrm{n}=3 /$ time point), and of male control-mice at one single time point $(n=5)$. In the following experiments cervical dislocation of male and female control-mice treated with $\mathrm{NaCl}$ alone were made at one single time point.

\section{Tissue preparation}

Brains were removed immediately, and either the whole brain was rapidly frozen on dry ice and stored at $-20^{\circ} \mathrm{C}$ or transferred to ice cold artificial cerebral fluid for dissection of leptomeninges and corpus callosum and then frozen on dry ice. The frozen tissue was later processed for Western blot and CAMP assay as described below. For in situ hybridization histochemistry and staining, $14 \mu \mathrm{m}$ thick coronal tissue sections of whole brain were cut on a microtome-cryostat (Microm HM500 OM, Walldorf, Germany), thaw-mounted onto 3aminopropyltriethoxysilane (Sigma-Aldrich)-coated slides, and stored at $-20^{\circ} \mathrm{C}$ until further processing.

\section{Estrous cycle determination}

A sample of cells present in female mice vagina $(n=17)$ was collected with the help of the tip of a plastic pipette filled with saline $(10 \mu \mathrm{l})$ at the day of experimentation. The tip was placed approximately $1 \mathrm{~mm}$ into the vagina and repeated flushes with the same solution, three to five times, were carried out. The final flush containing vaginal fluid was placed on a glass slide and visually observed under light microscope (Zeiss Axioplan, Zeiss, Oberkochen, Germany), the sample was then smeared over the slide and dried for staining with Diff-quick (with the kind help and scientific advice from Dr. Teresa Ribalta from Anatomía Patológica, Hospital Clínic Barcelona (Data not shown)).

\section{Hybridization probes}


The oligonucleotide probes complementary to the mRNAs coding for the different PDEs, inflammatory and cell markers have previously been published (Johansson et al., 2011). The mRNA regions selected for each PDE4B splice variant shared no similarities (Reyes-Irisarri et al., 2008). The hybridization conditions used to detect all mRNAs are described elsewhere (Miró et al., 2002;Pérez-Torres et al., 2000;Reyes-Irisarri et al., 2005). All oligonucleotides were synthesized and then purified by high-performance liquid chromatography (biomers.net $\mathrm{GmbH}, \mathrm{Ulm}$, Germany and Isogen Bioscience BV, Maarsden, The Netherlands). The specificity of the autoradiographic signal obtained in the in situ hybridization histochemistry experiments was confirmed by a series of routine controls similar to those previously described (Pompeiano et al., 1992).

Oligonucleotides were labeled at their $3^{\prime}$-end using $\left[\alpha^{-}{ }^{33} \mathrm{P}\right]$ dATP $(3000 \mathrm{Ci} / \mathrm{mmol}$, New England Nuclear, Boston, MA, USA) with terminal deoxynucleotidyl-transferase (TdT) (Calbiochem, Darmstadt, Germany). All vascular cell adhesion molecule-1 (VCAM-1), glial fibrillary acidic protein (GFAP), microtubule-associated protein (MAP2), myelin basic protein (MBP), and platelet-activating factor receptor (PAFR) oligonucleotides (100 pmol) were individually nonradioactively labeled with recombinant TdT (Roche Diagnostics $\mathrm{GmbH}$, Penzberg, Germany) and digoxigenin (DIG)-11-dUTP (Boehringer Mannheim, Mannheim, Germany) according to a previously described procedure (Schmitz et al., 1991). Labeled probes were purified using ProbeQuant G-50 Microcolumns (GE Healthcare, Buckinghamshire, UK).

\section{In situ hybridization histochemistry}

The protocols for single- and double-label in situ hybridization histochemistry were based on previously described procedures (Landry et al., 2000;Tomiyama et al., 1997) and have been published elsewhere (Reyes-Irisarri et al., 2007; Serrats et al., 2003).

For film autoradiography, hybridized sections were exposed to Biomax-MR (Kodak, Rochester, NY, USA) films for $2-20$ days at $-70{ }^{\circ} \mathrm{C}$ with intensifying screens. Double in situ hybridized sections were treated as described in (Landry et al., 2000). They were exposed in the dark at 4 ${ }^{\circ} \mathrm{C}$ for 6 weeks, developed in a Kodak D19 (Kodak) developer for $5 \mathrm{~min}$, and fixed in Ilford Hypam fixer (Ilford, Mobberly, Cheshire, UK).

\section{Analysis and preparation of figures from ISHH}

For single in situ hybridization experiments, semi-quantitative measurements of film optical densities were conducted using an AIS computerized image system (Imaging Research, St Catharines, Ontario, Canada). Sections were stained with cresyl-violet to identify brain structures with the aid of the Franklin and Paxinos Mouse Brain Atlas (Franklin and Paxinos, 2007). The optical densities corresponding to the following regions were measured on autoradiograms obtained from coronal tissue sections: Cornu ammonis fields (CA1-2, CA3), dentate gyrus (DG), hippocampal fissure (hf), subfornical organ (SFO), caudate putamen (CPu), cingulate cortex ( $\mathrm{Cg})$, nuclei of the inferior colliculus (IC) and leptomeninges (lepto.).

First, statistical comparisons using the factors brain region and treatment were carried out by separate two-way analyses of variance followed by post hoc Bonferroni's test for treatment and time for each brain region. Then, following normalization to $\mathrm{NaCl}$ administration in each region statistical comparisons using the factors sex and LPS administration were carried out by separate two-way ANOVA followed by post hoc Bonferroni's test for sex and time point for each brain region separately. All statistical analyses were performed using GraphPad Prism 4 (GraphPad Software, San Diego, CA, USA). Details of the analysis of double in situ hybridization experiments are described in Johansson et al. (2011).

Images from film autoradiograms were obtained using a Wild 420 macroscope (Leica Microsystems, Wetzlar, Germany) equipped with a digital camera (DXM1200 F, Nikon, Tokyo, Japan) and ACT-1 Nikon software. Microphotography was performed with an Olympus BX51 
Stereo Microscope (Olympus) equipped with a digital camera (DP71, Olympus, Tokyo, Japan). Figures were assembled using Adobe Photoshop (Adobe Systems, San Jose, CA, USA); only contrast and brightness were adjusted to optimize images.

\section{Fluoro-Jade B staining}

The protocol used has previously been published (Johansson et al., 2011).

\section{Western Blot}

Dissected tissue (corpus callosum and leptomeninges) from control and LPS injected (1h, $3 \mathrm{~h}$, $16 \mathrm{~h}$ and $24 \mathrm{~h}$ ) mouse brain were homogenized in ice-cold RIPA Buffer with Halt protease phosphates inhibitor cocktail (Thermo Scientific, Rockford, IL, USA). Homogenates from 6-10 animals from each time point after LPS administration and control were examined from two separate experiments.

The protein concentration of each pool was determined with Pierce ${ }^{\circledR}$ BCA Protein Assay Kit (Thermo scientific). 20-30 $\mu \mathrm{g}$ of total protein was loaded in each lane separated with $10-12 \%$ SDS-polyacrylamide gel electrophoresis and transferred to a nitrocellulose or PVDF membrane (Bio-Rad, Hercules, CA, USA). The membrane was blocked in $5 \%$ non-fat Milk or BSA in TBS / $0.1 \%$ Tween 20 (TBST) at room temperature for $1 \mathrm{~h}$. The antibodies used were: an anti-PDE4B polyclonal antibody C-terminal specific (FabGennix Inc., Frisco, TX, USA), anti-CREM (Abcam, Cambridge, UK) and the monoclonal antibodies: anti-CREB-1 (Abcam), anti-CREB phospho $\$ 133$ (Millipore, CA, USA) and anti-MBP (Abcam). All except for phosphor-CREB were diluted in TBST for protein immunoblot analysis and incubated for $1-2 \mathrm{~h}$ under agitation. Incubation with HRPconjugated anti-rabbit IgG (Jackson Immunoresearch Laboratories, PA, USA), anti-mouse IgG (Cell Signaling Technology, MA, USA) and anti-rat IgG (AbD Serotec, Oxford, UK) was performed for 1-2h. Specific protein bands were revealed with Immuno Star Chemiluminiscent Kit (Bio-Rad) or ECL Prime Western Blotting Detection Reagent (GE Healthcare, Buckinghamshire, UK). Stripping buffer was used to remove previous antibody staining from the membrane. Anti- $\beta$-actin (Sigma-Aldrich) immunoblotting was used as loading control. The membranes were scanned using Versadoc (Bio-Rad) and the resulting images were analyzed and quantified using the associated Quantity One software (Bio-Rad). The intensity of the immunoreactive bands from phospho-CREB and the co-migrating ICER-isoforms in each lane was corrected against the corresponding intensity of the CREB-stained band, while PDE4B and $M B P$ were corrected against $\beta$-actin. All experiments were analyzed as duplicates or triplicates, only values within the range of 2 standard deviations were included for further analysis. Statistical comparisons using the factors sex and LPS administration were carried out by separate two-way ANOVA followed by post hoc Bonferroni's test for sex and time point.

\section{cAMP Assay}

For cAMP assay, brain hemispheres from LPS administered or control mice were homogenized in 5\% trichloroacetic acid (TCA) in water (Calbiochem) and the TCA was extracted from the sample using water-saturated ether. Samples were stored at $-20^{\circ} \mathrm{C}$ until processed following day.

The effect of LPS administration on cAMP levels was studied using a cAMP Assay Kit (Cayman, Tallinn, Estonia) based on the competition between free CAMP and a CAMPacetylcholinesterase conjugate (cAMP-tracer) for a limited number of cAMP-specific rabbit antibody binding sites. Protocol and analysis were performed according to manufacturer's instructions. In brief, cAMP concentration (pg cAMP/ml) was calculated by interpolation to values (triplicates) determined with known concentrations of cAMP (standard curve) in the same assay. The CAMP standard was provided by the manufacturer. After normalization statistical comparisons using the factors sex and LPS administration were carried out by separate two-way ANOVA followed by post hoc Bonferroni's test for sex and time point. 


\section{RESULTS}

\section{Cell survival and neuroinflammatory response}

After staining with Fluoro-Jade $B$, a small number of necrotic cells were detected in the DG in both male and female (Fig. 1A-B), indicating that no severe effects on cell survival aroused from the LPS administration at this time point. No other brain region investigated showed positive staining. However, as described in previous studies from our laboratory (Johansson et al., 2011) a lethality test confirmed that a high LPS dose provoke septic shock at a later time point.
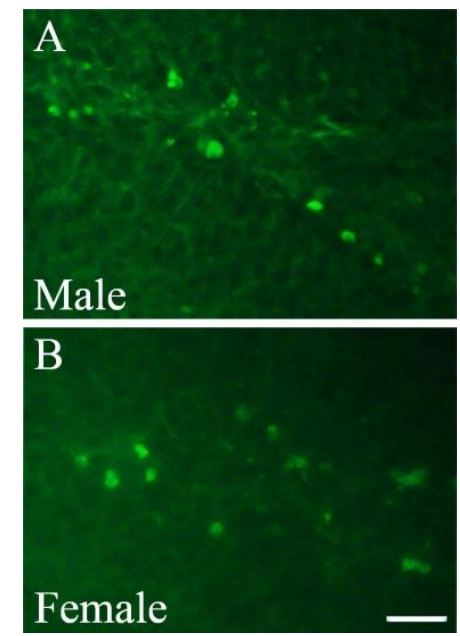

Figure 1. Presence of necrotic cells following LPS administration. Photomicrographs were taken from coronal sections of the dentate gyrus of animals sacrificed $24 \mathrm{~h}$ post-injection $(10 \mathrm{mg} / \mathrm{kg})$. Fluoro-Jade $B$ in male $(\mathbf{A})$ and female mouse brain (B). Scale bar $=100 \mu \mathrm{m}$.

We observed an innate neuroinflammatory response in our animal model upon analysis of mRNA alterations of the inflammatory markers; COX-2, IL-1 $\beta$ and VCAM-1 after LPS administration. GFAP mRNA expression was used to characterize activation of astrocytes and astrogliosis. By visually analyzing images from film autoradiograms a time-dependent response in mRNA hybridization levels were observed in both male and female. An increase in expression was observed $3 \mathrm{~h}$ after administration that declined after $24 \mathrm{~h}$ in both sexes for IL-1 $\beta$ and VCAM-1 mRNA (data not shown). The COX-2 mRNA expression also exhibited a common peak at this time point for both sexes (Fig. 2B, E) whereas a return to basal expression only could be observed in female after $24 \mathrm{~h}$ (Fig. 2C, F). A moderate upregulation of GFAP mRNA was observed $3 \mathrm{~h}$ after LPS administration in both sexes (Fig. $2 \mathrm{H}, \mathrm{K}$ ) and in contrast to COX-2, male showed stronger GFAP expression levels $24 \mathrm{~h}$ post-injection compared to female (Fig. 2l, L). 

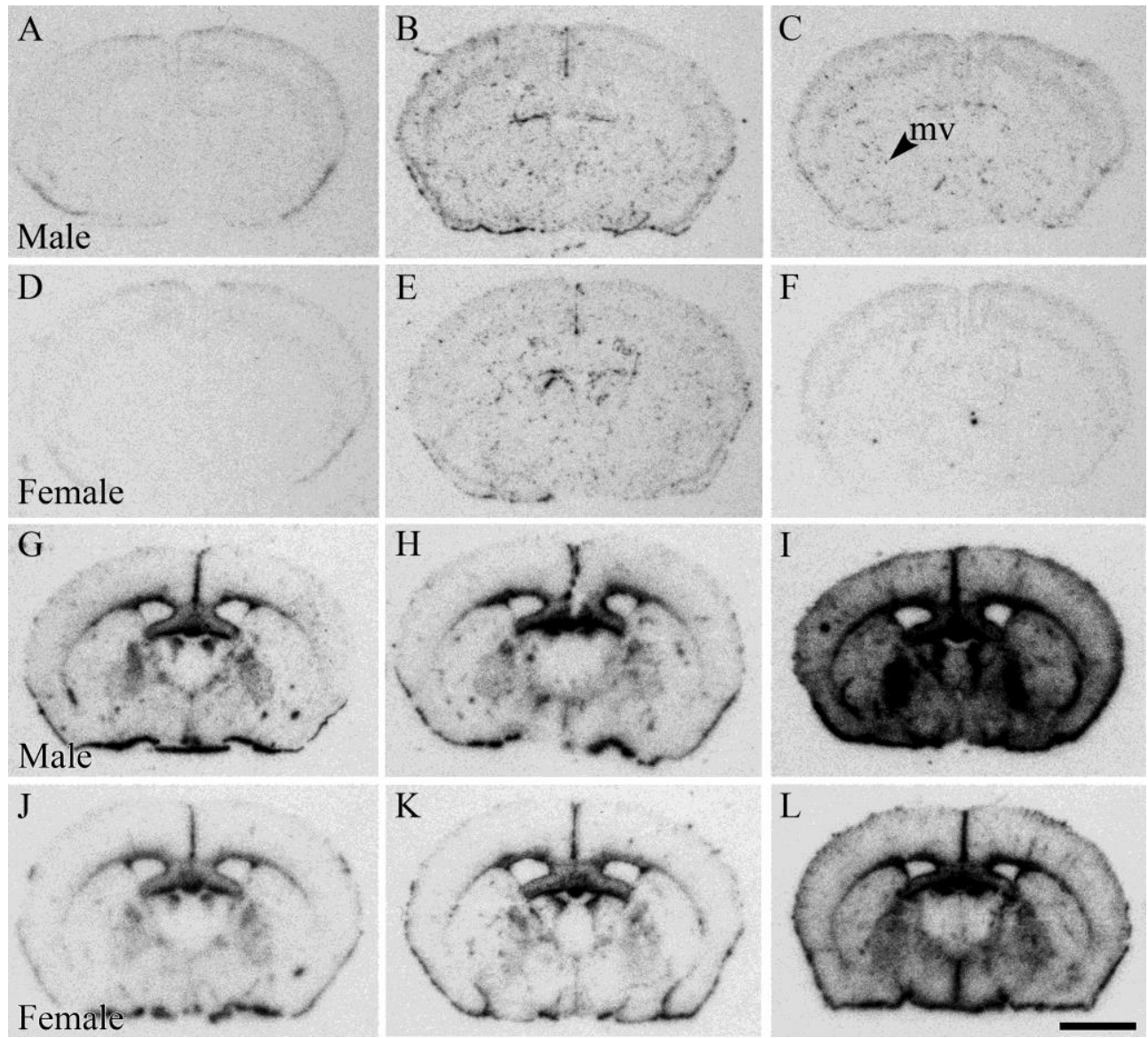

Figure 2. Expression of COX-2 and GFAP mRNAs in mouse brain following LPS administration. Macroscopic photographs of film autoradiograms showing the localization of the mRNAs coding for COX-2 (A-F) and GFAP (G-L) following i.p. LPS administration in mouse male and female coronal sections. COX-2 mRNA expression is prominent in microvessels (mv) $3 \mathrm{~h}$ after LPS administration in both sexes (B, E) and after $24 \mathrm{~h}$ in male (C) whereas in female a return to basal levels is observed at this time point $(\mathbf{F})$. Note the stronger GFAP mRNA hybridization levels in male compared to female $24 \mathrm{~h}$ after LPS (I, L). Control (A, D, G, J) injected saline; LPS (10mg/kg) at 3h (B, E, H, K) and $24 \mathrm{~h}$ $(C, F, I, L)$. COX-2, cyclooxygenase; GFAP, glial fibrillary acidic protein. Scale bar $=5 \mathrm{~mm}$.

\section{PDE4B2 and PDE4B3 mRNA expression in male and female following acute neuroinflammation}

We analyzed by microdensitometry the expression of CAMP-specific PDE4B splice variant mRNAs on film autoradiograms obtained after in situ hybridization in mouse brain, comparing male and female. The leptomeninges, SFO, IC (consisting of the external cortex, and the nucleus of the brachium of the inferior colliculus), Cg (anterior), the hippocampal fissure and the hippocampal formation (CA1-3 and DG) (Fig. 3A-C) were selected for measurement of their relative optical densities due to their close contact with the brain exterior and consequently their possible sensitivity during innate immediate neuroinflammation. We choose to measure the IC as an example of the parenchyma proximal to areas in close contact to the leptomeninges (Fig. 3C). Consistent with our prior work no alterations were noticed for the PDE4B1 or the PDE4B4 splice variant mRNAs expression after LPS administration in neither sex (results not shown). However, both PDE4B2 and PDE4B3 splice variants presented alterations in mRNA hybridization levels related to both LPS administration and to sex in a timedependent manner (Fig. 3). 

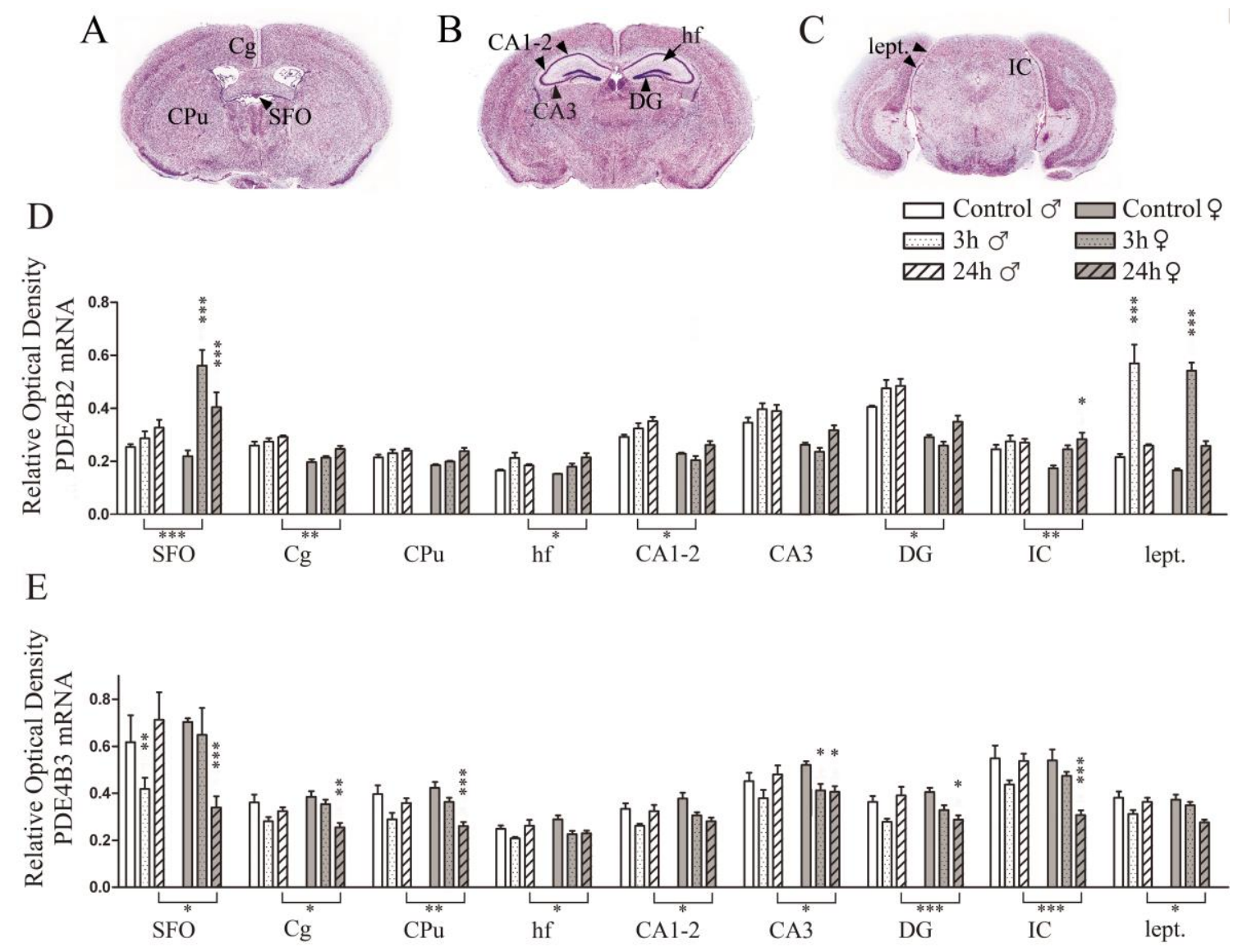

Figure 3. Alterations in expression of PDE4B2 and PDE4B3 mRNA in mouse brain following LPS administration. Effects of LPS administration $(10 \mathrm{mg} / \mathrm{kg})$ in different mouse brain areas $(A-C)$ on the mRNA expression of PDE4B splice variants PDE4B2 (D) and PDE4B3 (E) 3 and 24h post-injection in male and female. Note the opposite effects provoked by LPS on mRNA expression for the two splice variants and the significant difference in downregulation observed between male and female for the PDE4B3 mRNA after 24h. Furthermore, a significant downregulation of PDE4B3 mRNA expression is shown in the majority of female brain areas at $24 \mathrm{~h}$. Data are the mean \pm SEM $(n=5$ mice/group). Statistically significant differences between male and female groups after normalization are shown with brackets between bars and significant differences in relative optical densities between the LPS-stimulated and the control groups in different brain areas are both represented by ${ }^{*} P<0.05,{ }^{*} P<0.01, * * * P<0.001$; Bonferroni posttest. CA1-2, CA3, fields of Cornu ammonis; DG, dentate gyrus; hf, hippocampal fissure; SFO, subfornical organ; $\mathrm{Cg}$, cingulate cortex; $\mathrm{CPu}$, caudate putamen; IC, nuclei of the inferior colliculus; lept., leptomeninges.

No sex-related variations could be observed between vehicle-administered male and female mouse brains in basal PDE4B-splice variant mRNA levels, nor could any differences be noticed when comparing control female mice after different saline-treatment time points $(1 \mathrm{~h}, 3 \mathrm{~h}, 8 \mathrm{~h}$, $24 \mathrm{~h}$, and $48 \mathrm{~h}$ ) (results not shown). The clear difference between sexes in mRNA expression of PDE4B3 24h post-injection in all brain areas investigated (Fig. 3E) together with its abundant expression in whole brain in control animals (Fig. 4E, 5E) led us to determine the identity of the cells expressing this mRNA splice variant (neurons, oligodendrocytes and astrocytes) in the parenchyma (IC) (Fig. 3C) and Cg (Fig. 3A). Based on previous reports from our laboratory the expression of PDE4B3 mRNA in astrocytes was not investigated in the cingulate cortex (Johansson et al., 2011).

\section{PDE4B2 mRNA expression in male and female following acute neuroinflammation}

Under basal conditions the PDE4B2 mRNA expression was weak in whole mouse brain (Fig. 4A, E) except for the hippocampal formations. Already $1 \mathrm{~h}$ following immune challenge with LPS, upregulation of PDE4B2 mRNA levels were clearly observed in leptomeninges (data not shown) peaking around $3 \mathrm{~h}$ post-injection (Fig. 3D, 4B, 4F) in both sexes. This sharp upregulation was 
also observed 3h after injection in female SFO (Fig. 3D), while in male this appeared only after $8 \mathrm{~h}$ (Johansson et al., 2011). Significant upregulation was observed in IC in male after $8 \mathrm{~h}$ (Fig. 4C) and in female after 24h (Fig. 3D, 4G). Furthermore, a trend to increased intensity of PDE4B2 mRNA expression was observed in various brain areas e.g. the hippocampal areas CA12 and DG 3 h post-injection in male differing significantly from female were upregulation was observed after 24h (Fig. 3D). The hippocampal fissure, $\mathrm{Cg}$ and IC showed a tendency to return to control levels $24 \mathrm{~h}$ post-injection in male, revealing a significant difference compared to female which continued to show elevated expression (Fig. 3D, 4D, 4G) only returning to control levels around $48 \mathrm{~h}$ following LPS-administration (Fig. 4H). Caution must be taken when comparing the variations in the overall hybridization signal intensity of the same labeled oligonucleotide probe from different experiments, observed here for male and female.

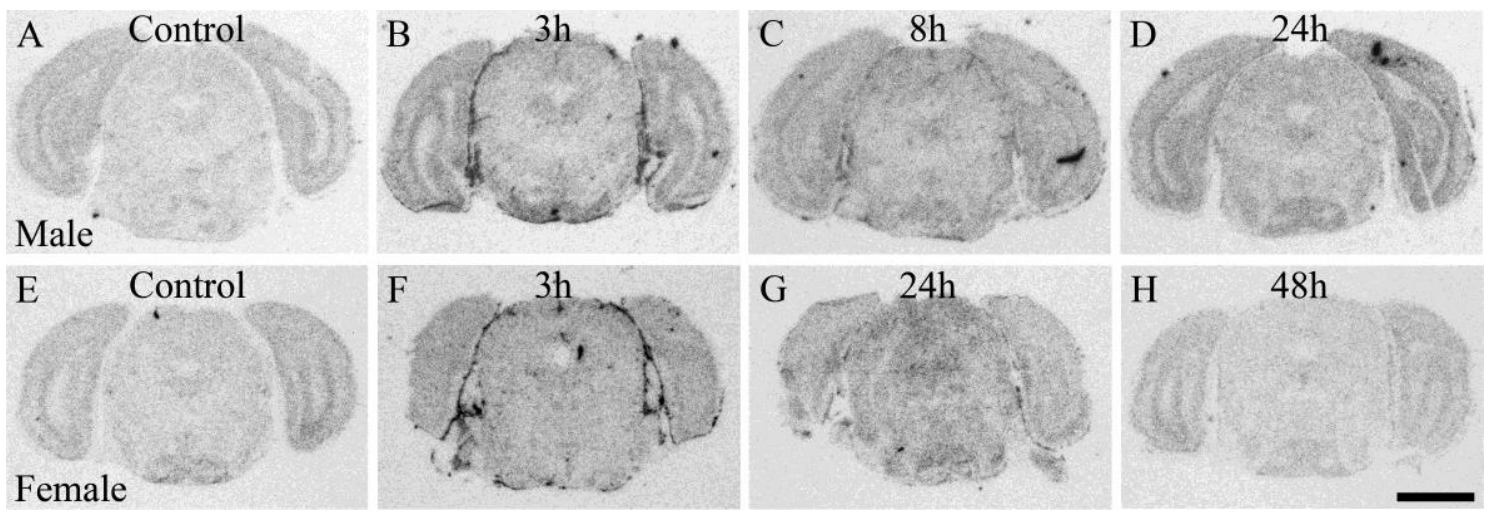

Figure 4. Expression of PDE4B2 mRNA following LPS administration in male and female brains. Macroscopic photographs of film autoradiographic images of mouse coronal sections showing alterations in mRNA hybridization signal of PDE4B2 in male (A-D) and female (E-H). Control animals (saline i.p.) (A, E); LPS treated animals $(10 \mathrm{mg} / \mathrm{kg}$ i.p): $3 \mathrm{~h}(\mathbf{B}, \mathbf{F}), 8 \mathrm{~h}(\mathbf{C}), 24 \mathrm{~h}(\mathbf{D}, \mathbf{G})$ and $48 \mathrm{~h}(\mathbf{H})$ after injection. Note the difference in time between male and female in the return to basal levels $(D, H)$. Scale bar $=5 \mathrm{~mm}$.

\section{PDE4B3 mRNA expression in male and female following acute neuroinflammation}

PDE4B3 mRNA is abundantly expressed throughout the brain under basal conditions and following LPS administration a downregulation in whole brain was revealed in contrast to the upregulation observed for PDE4B2 mRNA in specific brain areas. A significant decrease in the PDE4B3 mRNA was observed in female in almost all brain areas measured $24 \mathrm{~h}$ post-injection (Fig. 3E, 5G). Furthermore this decrease was also significantly different between sexes (Fig. 3E). Additionally, significant differences between sexes were observed in a time-dependent manner. An overall trend to PDE4B3 mRNA reduction was observed already 3 and 8 h postinjection in male (Fig 3E, 5B-C) whereas in female the minimum hybridization levels were attained $24 \mathrm{~h}$ after LPS administration (Fig. 3E, 5G). A return towards control densities was remarked $24 \mathrm{~h}$ post-injection in male (Fig. $3 \mathrm{E}, 5 \mathrm{D}$ ), and in female this was observed after $48 \mathrm{~h}$ (Fig. 5H).
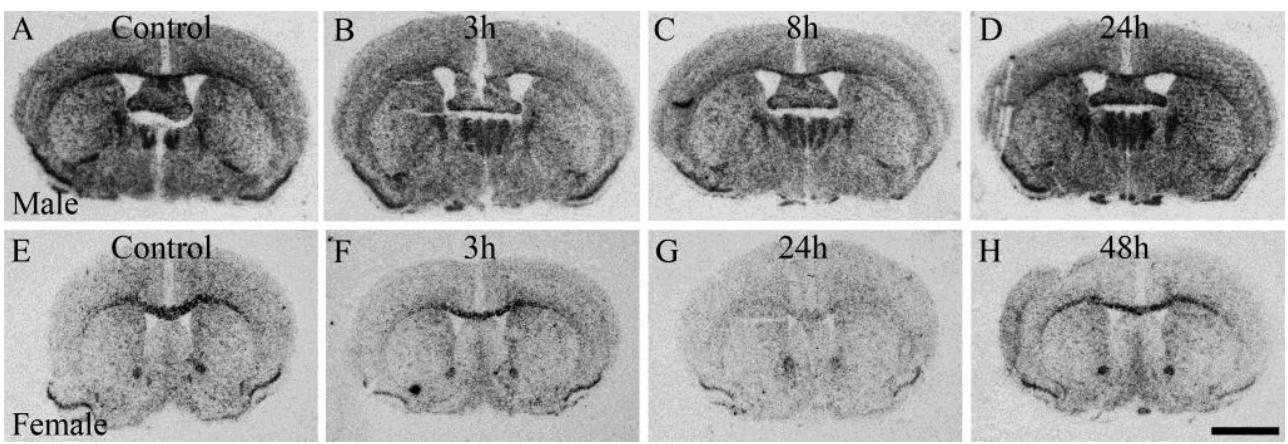

Figure 5. Expression of PDE4B3 mRNA following LPS administration in male and female brains. Macroscopic photographs of film autoradiographic images of mouse coronal sections showing alterations in mRNA hybridization 
signal of PDE4B3 in male (A-D) and female (E-H). Control animals (saline i.p.) (A, E); LPS treated animals (10mg/kg i.p): $3 \mathrm{~h}(\mathbf{B}, \mathbf{F}), 8 \mathrm{~h}(\mathbf{C}), 24 \mathrm{~h}(\mathbf{D}, \mathbf{G})$ and $48 \mathrm{~h}(\mathbf{H})$ after injection. Note the marked decrease in hybridization levels for PDE4B3 mRNA after $24 \mathrm{~h}(\mathbf{G})$ in female and the return to basal levels $48 \mathrm{~h}$ after LPS-induced immune response (H). Scale bar $=5 \mathrm{~mm}$.

\section{Characterization of cells presenting LPS-altered expression of PDE4B2 and PDE4B3 mRNAs in male and female}

The augmented PDE4B2 mRNA expression was associated with an increase in the inflammatory cell populations: VCAM mRNA-positive (endothelial cells) and PAFR-mRNA positive (microglial/macrophage cells) (data not shown), confirming previous studies from our laboratory (Johansson et al., 2011).

Oligodendrocytes, positive for the mRNA coding for MBP, abundantly (around $70-80 \%$ ) expressed the PDE4B3 splice variant in IC under basal conditions in both sexes (Table 1). The downregulation observed for the PDE4B3 mRNA post-injection was localized to oligodendrocytes with significantly lower expression after $3 \mathrm{~h}$ in $\mathrm{Cg}$ in male $(\approx 55 \%)$ and after $24 \mathrm{~h}$ in both $\mathrm{Cg}$ and parenchyma (IC) in female ( $\approx 55,66 \%$ ) (Table 1 and Fig. 6B, 6F). The number of MBP positive cells counted was constant between control and LPS injected animals and western blot studies further confirmed the absence of changes in the amount of MBP following LPS administration (data not shown). Figure 6 shows an example of the coexpression observed in parenchyma between MBP mRNA positive cells and PDE4B3 mRNA in male and female following a high LPS dose. Analysis of the presence of PDE4B3 mRNA in the neuronal cell population (MAP2 mRNA-positive cells) showed that a relatively constant percentage of neurons in both regions expressed this $\mathrm{MRNA}$, with no distinction between sexes or time points after LPS injection (Table 1). Following LPS administration PDE4B3 splice variant mRNA was also expressed in astrocytes (GFAP mRNA positive cells) (around 30-60\%) exhibiting no sex-related differences (Table 1).

Table 1. Quantification of the presence of PDE4B3 mRNA in different cellular populations

\begin{tabular}{|c|c|c|c|c|c|c|c|c|c|c|c|c|}
\hline & \multicolumn{6}{|c|}{ Cingulate Cortex } & \multicolumn{6}{|c|}{ Nuclei of the inferior colliculus } \\
\hline & \multicolumn{3}{|c|}{ Male } & \multicolumn{3}{|l|}{ Female } & \multicolumn{3}{|c|}{ Male } & \multicolumn{3}{|l|}{ Female } \\
\hline & Control & $3 \mathbf{h}$ & $24 h$ & Control & $3 \mathbf{h}$ & $24 \mathrm{~h}$ & Control & 3h & $24 \mathrm{~h}$ & Control & 3h & $24 h$ \\
\hline MAP & $35 \pm 14$ & $43 \pm 1$ & $38 \pm 8$ & $46 \pm 2$ & $41 \pm 17$ & $39 \pm 7$ & $44 \pm 8$ & $35 \pm 13$ & $50 \pm 12$ & $36 \pm 8$ & $43 \pm 7$ & $43 \pm 11$ \\
\hline $\begin{array}{l}\text { MBP } \\
\text { GFAP }\end{array}$ & $80 \pm 3$ & $55 \pm 13^{* *}$ & $73 \pm 9$ & $83 \pm 7$ & $80 \pm 6$ & $55 \pm 15^{* \star \star}$ & $\begin{array}{l}71 \pm 1 \\
N D\end{array}$ & $\begin{array}{l}53 \pm 6 \\
53 \pm 14\end{array}$ & $\begin{array}{l}78 \pm 9 \\
33 \pm 13\end{array}$ & $\begin{array}{l}86 \pm 10 \\
N D\end{array}$ & $\begin{array}{l}76 \pm 4 \\
45+6\end{array}$ & $\begin{array}{l}66 \pm 5 \text { * } \\
45+10\end{array}$ \\
\hline
\end{tabular}

Quantification was performed in cingulate cortex and nuclei of the inferior colliculus of male and female mice sacrificed 3 and $24 \mathrm{~h}$ after intra peritoneal LPS administration $(10 \mathrm{mg} / \mathrm{kg})$ and control animals. Data are the mean \pm SD of 3-5 animals and represent the percentage of counted cells, neurons (MAP2), oligodendrocytes (MBP) and astrocytes (GFAP) expressing PDE4B3 mRNA. Each percentage was determined from a mean of 57.7 cells per animal, except GFAP positive cells in the cingulate cortex and nuclei of the inferior colliculus of control animals where no astrocytes were detected (5999 cells counted). ND, not detected. Statistically significant differences between the LPS-stimulated and the control groups in the two brain areas in both male and female are represented by $* P<0.05, * * P<0.01, * * * P<0.001$; Bonferroni posttest. 


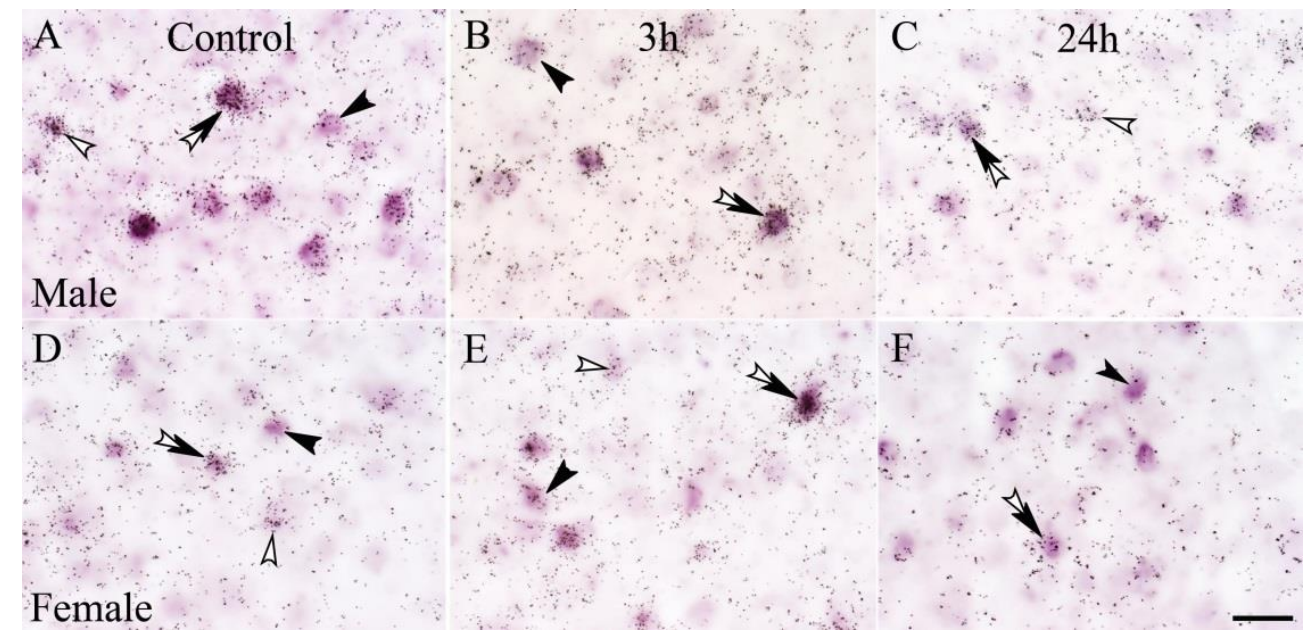

Figure 6. LPS-altered expression of PDE4B3 mRNA in oligodendrocytes. Cellular localization of PDE4B3 mRNA in oligodendrocytes in male (A-C) and female (D-F) mouse brain parenchyma corresponding to the area of nuclei of the inferior colliculus (IC). High-magnification bright-field microphotographs of emulsion-dipped sections, simultaneously showing mRNA visualized by double in situ hybridization using ${ }^{33} \mathrm{P}$-labeled oligonucleotides complementary to the mRNA coding for PDE4B3 (cluster of dark silver grains), with DIG-labeled oligonucleotides (dark precipitate) for MBP mRNA (oligodendrocytes). Control animals (A, D) and LPS treated animals $3 \mathrm{~h} \mathrm{(B,} \mathrm{E)} \mathrm{and}$ $24 \mathrm{~h}(\mathbf{C}, \mathbf{F})$ after injection. Black arrowheads point to digoxigenin-labeled cells, white arrowheads to radioactivelylabeled cells and double black and white arrowheads to double-labeled cells. Bar $=20 \mu \mathrm{m}$.

\section{Protein expression in male and female following acute neuroinflammation}

No changes were observed in PDE4B2 or PDE4B3 protein levels neither in male nor in female mouse leptomeninges or corpus callosum extracts at any time point following LPS administration (data not shown). The ICER protein expression showed a trend to decrease already $16 \mathrm{~h}$ post injection in females being significantly different between sexes after $24 \mathrm{~h}$ in corpus callosum (Fig.7B, C). Noteworthy is the decrease in variability observed at both $16 \mathrm{~h}$ and $24 \mathrm{~h}$ in female compared to all other time points in both sexes (Fig. 7C).

Phosphorylated CREB protein levels presented a similar expression in both male and female corpus callosum following innate immediate neuroinflammation (data not shown).

A

B

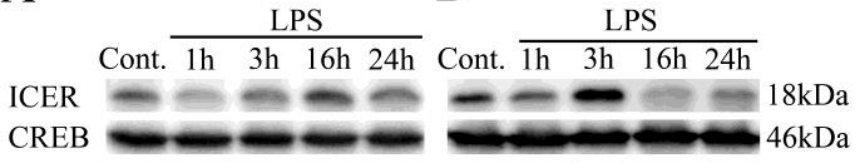

$\mathrm{C}$

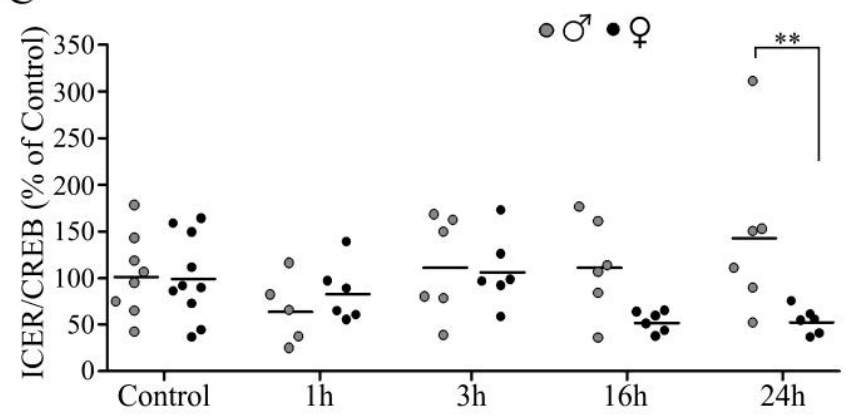

Figure 7. Alterations in ICER protein expression following LPS administration. Effects of LPS administration on the protein expression of ICER in male (A), and female (B) mouse brain dissection of corpus callosum 1, 3, 16 and $24 \mathrm{~h}$ post-injection and control. Graph shows results expressed as percentage of the control protein expression (C). Lines represent mean from 2-3 separate experiments with homogenates from each time point, animals are presented as grey (male) or black (female) dots ( $n=6-10$ mice/group). Statistically significant differences between male and female are represented by ${ }^{*} P<0.01$; Bonferroni posttest. 
Sex-related differences in CAMP levels from whole brain extracts were significant at several time points post-injection. In male a trend to higher concentration was noticed, reaching maximal levels around $32 \mathrm{~h}$ post-injection, whereas female showed a decrease at all time points following LPS administration (Fig. 8).

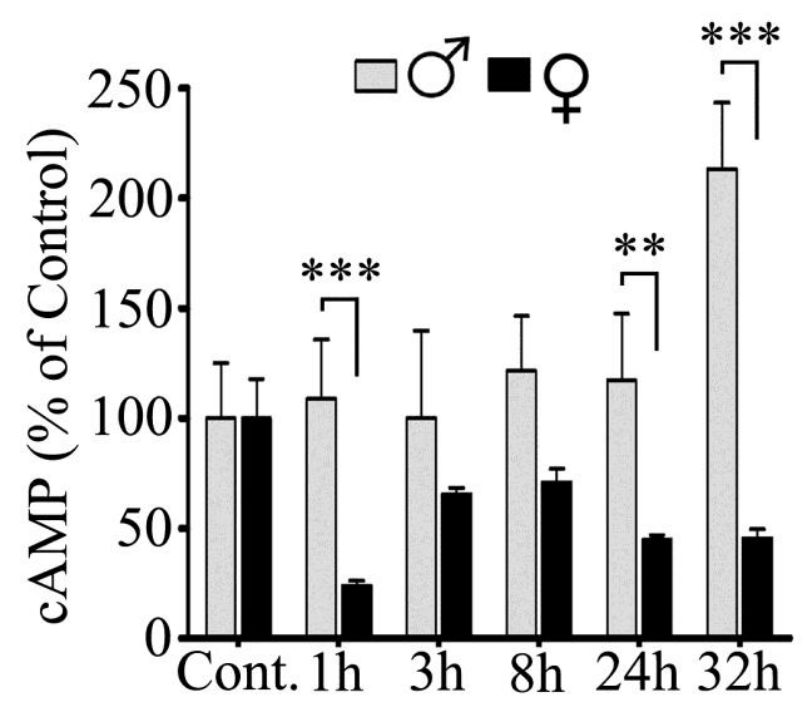

Figure 8. Alterations in cAMP levels in brain following LPS administration. Effects of LPS administration on the cAMP levels from male and female mouse brain 1, 3, 8, 24 and $32 \mathrm{~h}$ post-injection and control. Results are expressed as percentage of control cAMP levels from half brain homogenates. Values are mean \pm SD of 3 to 8 animals per time point, analyzed as triplicates. Statistically significant differences between male and female are represented by $* * P<0.01$ and $* * * P<0.001$; Bonferroni posttest.

\section{DISCUSSION}

This study deals with the importance of sex differences in brain research and endorses previous reports of sexual dimorphism in the innate inflammatory response. We aimed to determine whether there where basic sex differences in the alterations previously revealed in PDE mRNA expression in an animal model of innate neuroinflammation.

The possible influence of the reproductive cycle of the females used in the present work on the PDE4B splice variants expression was excluded considering the following: i) No alterations of PDE4B mRNAs were observed at different time points in control females (from $1 \mathrm{~h}$ until $48 \mathrm{~h}$ ). ii) Similar variability of the data was obtained in male and female specimen. iii) We could discern different stages of the estrous cycle for the females investigated via vaginal smears. Together, these factors suggest that the reproductive state probably has no influence on the results presented here, as recently proposed (McCarthy et al., 2012). Given the fact that the in vivo experiments were conducted during daytime we can exclude the influence of the circadian cycle in the expression levels of PDE4B splice variants as described by Kim and coworkers (Kim et al., 2007).

We found that the PDE4B splice variants mRNA expression differ in male and female brains during an innate immune response induced by a high peripheral LPS dose, revealing a resemblance to variations normally reported for inflammatory markers. The elevated expression observed for COX-2 mRNA in males and not in females $24 \mathrm{~h}$ post-injection confirms the generally higher production of cytokines and COX-2 reported in male mice (Ashdown et al., 2007; Marriott et al., 2006). Furthermore, higher GFAP mRNA expression suggests a stronger 
innate immune response in males in accordance with the reported sex-related differences in severity of sepsis (Marriott et al., 2006;Schroder et al., 1998). The timing of the PDE4B2 and PDE4B3 mRNA responses show sexual dimorphism as male mice present a peak in PDE4B2 expression around $8 \mathrm{~h}$ in specific brain areas compared to the female peak observed after $24 \mathrm{~h}$. This, together with the return to control expression levels for both mRNAs, which in females occurs later than in males, agree with a later cytokine response in female mice (Gregory et al., 2000). Although the cytokine response during inflammation clearly differs between male and female, the differences in intensity and timing reported throughout the literature depend on many factors, including the disease model, species, area of investigation (Klingstrom et al., 2008;Murakami and Ono, 1987; Pitychoutis et al., 2009;Santos-Galindo et al., 2011). The higher numbers of the toll-like receptor (TLR)-4 - CD14 complex on macrophage cell surface and of LPS binding protein present during basal conditions in the male brain (Marriott et al., 2006) might result in the earlier inflammatory response to LPS administration we described here in male mice brain.

PDE4B2 mRNA is mainly localized in microglia and endothelial cells and PDE4B3 mRNA is expressed in oligodendrocytes (Olgs) and neurons, as we have previously described (Johansson et al., 2011). Olgs are known to be sensitive to cytokine release showing apoptosis and myelin damage (Cai et al., 2003;D'Souza et al., 1996) and, a decline in the oligodendrocytic cell population might provoke the observed downregulation of PDE4B3 transcript in these cells. However, a more plausible explanation might be by repression of PDE4B3 transcription since i) no apoptotic or necrotic cells were found, and ii) the amount of MBP-mRNA positive cells and the MBP protein expression were preserved following LPS administration. The low expression of TLR-4 on Olgs suggests (Lehnardt et al., 2002) that other pathways activated by inflammatory cytokines might be responsible for such transcriptional repression.

ICER is a potent endogenous repressor of CRE-mediated gene transcription, ubiquitously expressed in glia (Kell et al., 2004) and is therefore, a possible candidate responsible for the LPS induced PDE4B3 mRNA downregulation. However, in contrast to our working hypothesis that an increase in ICER would lead to repression of PDE4B3 transcription, we observed a decrease in ICER protein expression in female. The decrease in variability observed in ICER protein expression in females further supports a connection between LPS and ICER, comparable to that observed between LPS and PDE4B3 mRNA expression. Furthermore, no alterations were observed in the two PDE4B splice variants protein expression in either sex, suggesting higher PDE activity in female compared to male mice, coherent with the decreased CAMP concentrations observed in females. Decreased ICER expression might reflect a systematic attempt to increase PDE4B3 expression as a mean to recover the low cAMP levels, a possible explanation that deserves further investigation.

The sex hormones, estrogens and testosterone are thought to mediate several of the sex related differences in the immune response (Fairweather et al., 2008;Marriott et al., 2006). Interestingly, the promoters of typical proinflammatory cytokine genes lack estrogen response elements and alternative explanations have been suggested for estrogen-dependent inhibition of cytokine transcription (Pottratz et al., 1994). For example, tumor necrosis factor- $\alpha$ (TNF- $\alpha$ ) inhibition provoked by modulation of the AP-1 promoter has been explained by an estrogendependent decrease in upstream regulators of AP-1 binding (Srivastava et al., 1999). On the other hand, TNF signaling has been proven to activate the transcription factor AP-1 in glial cells (Rothe et al., 1994). Studies on the PDE4D genome revealed the presence of AP-1 binding site in the PDE4 promoter (Vicini and Conti, 1997) leaving both the estrogen and cytokines as other plausible regulators of the LPS-induced PDE4B mRNA alterations worth investigating.

The decrease in cAMP levels we observed in females, is relevant in view of the participation of this second messenger in the remyelization process that takes place following white matter damage as observed in multiple sclerosis (for review see (Chari, 2007)) and also in the 
protection of myelin from exocytosis (Yoshioka et al., 1998). In conclusion, we propose that the endogenous PDE4B3 mRNA downregulation observed in female brain could influence the effect of the acute neuroinflammation on oligodendrocytes, providing a possible explanation to their differential susceptibility to demyelinating diseases.

The extensive variation between sexes in the inflammatory response underlines the importance of sex-related investigation in order to understand the biology of neurological diseases in women and to improve pharmacological treatments of such diseases. It is also worth considering that many biochemical sex differences observed might be a reflection of a compensatory mechanism that ultimately makes males and females more similar (De Vries, 2004). The results presented here imply that manipulation of PDE activity requires sex specific investigation and further emphasize the issue of sexual bias in animal research studies.

\section{Acknowledgements}

This work was supported by grants awarded by the Spanish Ministerio de Educación y Ciencia (SAF2006-10243); Ministerio de Ciencia e Innovación (SAF 2009-11052; PI-10/01874), FEDER Funds (European Union) and by the Generalitat de Catalunya (SGR2009/220). Emily Johansson was a recipient of a fellowship from the Ministerio de Educación y Ciencia and Stiftelsen Olle Engkvist Byggmästare and Cristina Sanabra from the IDIBAPS and CIBERNED.

We thank Rocío Martín for technical assistance, Emanuela Santini for technical advice about Western Blot and Sairica Rose for English corrections.

\section{References}

Ansar AS, Penhale WJ, Talal N (1985) Sex hormones, immune responses, and autoimmune diseases. Mechanisms of sex hormone action. Am J Pathol 121: 531-551.

Arp J, Kirchhof MG, Baroja ML, Nazarian SH, Chau TA, Strathdee CA, Ball EH, Madrenas J (2003) Regulation of T-cell activation by phosphodiesterase 4B2 requires its dynamic redistribution during immunological synapse formation. Mol Cell Biol 23: 8042-8057.

Ashdown H, Poole S, Boksa P, Luheshi GN (2007) Interleukin-1 receptor antagonist as a modulator of gender differences in the febrile response to lipopolysaccharide in rats. Am J Physiol Regul Integr Comp Physiol 292: R1667-R1674.

Avni D, Ernst O, Philosoph A, Zor T (2010) Role of CREB in modulation of TNFalpha and IL-10 expression in LPS-stimulated RAW264.7 macrophages. Mol Immunol 47: 1396-1403.

Banner KH, Trevethick MA (2004) PDE4 inhibition: a novel approach for the treatment of inflammatory bowel disease. Trends in Pharmacological Sciences 25: 430-436.

Bender AT, Beavo JA (2006) Cyclic nucleotide phosphodiesterases: Molecular regulation to clinical use. Pharmacological Reviews 58: 488-520.

Borlikova G, Endo S (2009) Inducible cAMP early repressor (ICER) and brain functions. Mol Neurobiol 40: 73-86.

Cai Z, Pang Y, Lin S, Rhodes PG (2003) Differential roles of tumor necrosis factor-alpha and interleukin-1 beta in lipopolysaccharide-induced brain injury in the neonatal rat. Brain Res 975: 37-47.

Chari DM (2007) Remyelination in multiple sclerosis. Int Rev Neurobiol 79: 589-620.

Conti M (2000) Phosphodiesterases and cyclic nucleotide signaling in endocrine cells. Mol Endocrinol 14: 1317-1327.

Czlonkowska A, Ciesielska A, Gromadzka G, Kurkowska-Jastrzebska I (2005) Estrogen and cytokines production - the possible cause of gender differences in neurological diseases. Curr Pharm Des 11: 10171030.

D'Sa C, Tolbert LM, Conti M, Duman RS (2002) Regulation of cAMP-specific phosphodiesterases type 4B and 4D (PDE4) splice variants by cAMP signaling in primary cortical neurons. J Neurochem 81: 745-757. 
D'Souza SD, Alinauskas KA, Antel JP (1996) Ciliary neurotrophic factor selectively protects human oligodendrocytes from tumor necrosis factor-mediated injury. J Neurosci Res 43: 289-298.

Darnall BD, Suarez EC (2009) Sex and gender in psychoneuroimmunology research: past, present and future. Brain Behav Immun 23: 595-604.

De Vries GJ (2004) Minireview: Sex differences in adult and developing brains: compensation, compensation, compensation. Endocrinology 145: 1063-1068.

Fairweather D, Frisancho-Kiss S, Rose NR (2008) Sex differences in autoimmune disease from a pathological perspective. Am J Pathol 173: 600-609.

Franklin KBJ, Paxinos G (2007) The Mouse Brain in stereotaxic coordinates. Academic Press.

Gregory MS, Faunce DE, Duffner LA, Kovacs EJ (2000) Gender difference in cell-mediated immunity after thermal injury is mediated, in part, by elevated levels of interleukin-6. J Leukoc Biol 67: 319-326.

Johansson EM, Sanabra C, Cortes R, Vilaro MT, Mengod G (2011) Lipopolysaccharide administration in vivo induces differential expression of CAMP-specific phosphodiesterase $4 B$ mRNA splice variants in the mouse brain. J Neurosci Res 89: 1761-1772.

Kawai T, Takeuchi O, Fujita T, Inoue J, Muhlradt PF, Sato S, Hoshino K, Akira S (2001) Lipopolysaccharide stimulates the MyD88-independent pathway and results in activation of IFN-regulatory factor 3 and the expression of a subset of lipopolysaccharide-inducible genes. J Immunol 167: 5887-5894.

Kell CA, Dehghani F, Wicht H, Molina CA, Korf HW, Stehle JH (2004) Distribution of transcription factor inducible cyclicAMP early repressor (ICER) in rodent brain and pituitary. J Comp Neurol 478: 379-394.

Kim JS, Bailey MJ, Ho AK, Moller M, Gaildrat P, Klein DC (2007) Daily rhythm in pineal phosphodiesterase (PDE) activity reflects adrenergic/3',5'-cyclic adenosine 5'-monophosphate induction of the PDE4B2 variant. Endocrinology 148: 1475-1485.

Klingstrom J, Lindgren T, AhIm C (2008) Sex-dependent differences in plasma cytokine responses to hantavirus infection. Clin Vaccine Immunol 15: 885-887.

Landry M, Holmberg K, Zhang X, Hokfelt T (2000) Effect of axotomy on expression of NPY, galanin, and NPY Y1 and Y2 receptors in dorsal root ganglia and the superior cervical ganglion studied with doublelabeling in situ hybridization and immunohistochemistry. Exp Neurol 162: 361-384.

Lehnardt S, Lachance C, Patrizi S, Lefebvre S, Follett PL, Jensen FE, Rosenberg PA, Volpe JJ, Vartanian T (2002) The toll-like receptor TLR4 is necessary for lipopolysaccharide-induced oligodendrocyte injury in the CNS

1. J Neurosci 22: 2478-2486.

Lu YC, Yeh WC, Ohashi PS (2008) LPS/TLR4 signal transduction pathway

1. Cytokine 42: 145-151.

Marriott I, Bost KL, Huet-Hudson YM (2006) Sexual dimorphism in expression of receptors for bacterial lipopolysaccharides in murine macrophages: a possible mechanism for gender-based differences in endotoxic shock susceptibility. J Reprod Immunol 71: 12-27.

McCarthy MM, Arnold AP, Ball GF, Blaustein JD, De Vries GJ (2012) Sex differences in the brain: the not so inconvenient truth. J Neurosci 32: 2241-2247.

Miró X, Perez-Torres S, Artigas F, Puigdomenech P, Palacios JM, Mengod G (2002) Regulation of cAMP phosphodiesterase mRNAs expression in rat brain by acute and chronic fluoxetine treatment. An in situ hybridization study. Neuropharmacology 43: 1148-1157.

Moore AR, Willoughby DA (1995) The role of cAMP regulation in controlling inflammation. Clin Exp Immunol 101: 387-389.

Moreno B, Jukes JP, Vergara-Irigaray N, Errea O, Villoslada P, Perry VH, Newman TA (2011) Systemic inflammation induces axon injury during brain inflammation. Ann Neurol 70: 932-942.

Murakami N, Ono T (1987) Sex-related differences in fever development of rats. Am J Physiol 252: R284R289. 
Pérez-Torres S, Miró X, Palacios JM, Cortés R, Puigdoménech P, Mengod G (2000) Phosphodiesterase type 4 isozymes expression in human brain examined by in situ hybridization histochemistry and $[3 \mathrm{H}]$ rolipram binding autoradiography. Comparison with monkey and rat brain. Journal of Chemical Neuroanatomy 20: 349-374.

Pitychoutis PM, Nakamura K, Tsonis PA, Papadopoulou-Daifoti Z (2009) Neurochemical and behavioral alterations in an inflammatory model of depression: Sex differences exposed. Neuroscience 159: 12161232.

Pompeiano M, Palacios JM, Mengod G (1992) Distribution and cellular localization of mRNA coding for 5HT1A receptor in the rat brain: correlation with receptor binding. J Neurosci 12: 440-453.

Pottratz ST, Bellido T, Mocharla H, Crabb D, Manolagas SC (1994) 17 beta-Estradiol inhibits expression of human interleukin-6 promoter-reporter constructs by a receptor-dependent mechanism. J Clin Invest 93: 944-950.

Reyes-Irisarri E, Perez-Torres S, Mengod G (2005) Neuronal expression of cAMP-specific phosphodiesterase 7b mRNA in the rat brain. Neuroscience 132: 1173-1185.

Reyes-Irisarri E, Perez-Torres S, Miro X, Martinez E, Puigdomenech P, Palacios JM, Mengod G (2008) Differential distribution of PDE4B splice variant mRNAs in rat brain and the effects of systemic administration of LPS in their expression. Synapse 62: 74-79.

Reyes-Irisarri E, Sanchez AJ, Garcia-Merino JA, Mengod G (2007) Selective induction of cAMP phosphodiesterase PDE4B2 expression in experimental autoimmune encephalomyelitis. Journal of Neuropathology and Experimental Neurology 66: 923-931.

Rothe M, Wong SC, Henzel WJ, Goeddel DV (1994) A novel family of putative signal transducers associated with the cytoplasmic domain of the 75 kDa tumor necrosis factor receptor. Cell 78: 681-692.

Santos-Galindo M, Acaz-Fonseca E, Bellini MJ, Garcia-Segura LM (2011) Sex differences in the inflammatory response of primary astrocytes to lipopolysaccharide. Biol Sex Differ 2: 7.

Schmitz GG, Walter T, Seibl R, Kessler C (1991) Nonradioactive labeling of oligonucleotides in vitro with the hapten digoxigenin by tailing with terminal transferase. Anal Biochem 192: 222-231.

Schroder J, Kahlke V, Staubach KH, Zabel P, Stuber F (1998) Gender differences in human sepsis. Arch Surg 133: 1200-1205.

Serrats J, Artigas F, Mengod G, Cortes R (2003) GABAB receptor mRNA in the raphe nuclei: co-expression with serotonin transporter and glutamic acid decarboxylase. J Neurochem 84: 743-752.

Srivastava S, Weitzmann MN, Cenci S, Ross FP, Adler S, Pacifici R (1999) Estrogen decreases TNF gene expression by blocking JNK activity and the resulting production of c-Jun and JunD. J Clin Invest 104: 503-513.

Tomiyama M, Palacios JM, Cortes R, Vilaro MT, Mengod G (1997) Distribution of AMPA receptor subunit mRNAs in the human basal ganglia: an in situ hybridization study. Brain Res Mol Brain Res 46: 281-289.

Vicini E, Conti M (1997) Characterization of an intronic promoter of a cyclic adenosine 3',5'monophosphate (CAMP)-specific phosphodiesterase gene that confers hormone and cAMP inducibility. Mol Endocrinol 11: 839-850.

Yoshioka A, Shimizu Y, Hirose G, Kitasato H, Pleasure D (1998) Cyclic AMP-elevating agents prevent oligodendroglial excitotoxicity. J Neurochem 70: 2416-2423. 